\title{
A Spatial Study of X-ray Properties in Superbubble 30 Dor C with XMM-Newton
}

\author{
Babazaki, Y. ${ }^{1}$, Mitsuishi, I. ${ }^{1}$, Sano, H. ${ }^{1}$, Yoshiike, S. ${ }^{1}$, Fukuda, T. ${ }^{1}$, \\ Maruyama, S. ${ }^{1}$, Fujii, K. ${ }^{1}$, Fukui, Y. ${ }^{1}$, Tawara,.$^{1}$ and Matsumoto, $\mathbf{H}^{2}$ \\ ${ }^{1}$ Department of Physics, Nagoya University, Furo-cho, Chikusa-ku, Nagoya 464-8601, Japan; \\ email: y_babazaki@u.phys.nagoya-u.ac.jp \\ ${ }^{2}$ Kobayashi-Maskawa Institute (KMI), Nagoya University, Furo-cho, Chikusa-ku, Nagoya, \\ Aichi, Japan 464-8602
}

\begin{abstract}
The superbubble (SB) 30 Dor C with the strong non-thermal X-ray emission is one of the best targets for study of the cosmic-ray (CR) acceleration. We investigated X-ray spectral properties of the SB with a high spatial resolution of $\sim 10 \mathrm{pc}$. Consequently, the spectra in the east regions can be described with a combination of absorbed thermal and non-thermal models while the spectra in the west regions can be fitted with an absorbed non-thermal model. We found that the observed photon index and intensity in 2-10 keV show variations of 2.0-3.5 and (0.6-8.0) $\times 10^{-7} \mathrm{erg} \mathrm{s}^{-1} \mathrm{~cm}^{-2} \mathrm{str}^{-1}$, respectively. The results are possibly caused by the spatial variation of the $\mathrm{CR}$ acceleration efficiency and/or the circumstellar environment.
\end{abstract}

Keywords. cosmic rays - ISM: supernova remnants - ISM: bubbles - X-rays: ISM

Sano et al. (2015) detected the sub-parsec spatial variations of the non-thermal Xray properties in SNR RX J1713.7-3946 and a correlation between the X-ray intensity and the molecular mass interacting with the SNR. To explain the observational results, they suggested the shock-cloud interaction scenario (see the details [1]). Thus, in this work, we investigate X-ray properties of a superbubble (SB) which possesses a larger spatial extent created by successive supernova explosions and aim at constructing a CR acceleration mechanism also for SBs. 30 Dor C, located in Large Magellanic Cloud, is a young SB showing a shell structure with a diameter of $\sim 80 \mathrm{pc}\left(\sim 6^{\prime}\right)$ in X-ray and bright non-thermal X-ray luminosity of $\sim 5 \times 10^{35} \mathrm{erg} \mathrm{s}^{-1}$ [2]. Recently, TeV $\gamma$-ray emission associated with the SB was detected for the first time [3], meaning that CR protons/electrons are efficiently accelerated up to at least $10 \mathrm{TeV}$.

We used XMM-newton EPIC-pn datasets and divided the X-ray shell into 33 regions with $0.7^{\prime} \times 0.7^{\prime}$ grids corresponding to a physical scale of $\sim 10 \mathrm{pc}$ in order to examine the detailed X-ray properties in the SB. Consequently, the spectra in the east regions of 30 Dor $\mathrm{C}$ can be described with absorbed thermal and non-thermal models, while the spectra in the west regions can be fitted with an absorbed non-thermal model. The observed photon index and intensity in 2-10 keV show spatial variations of 2.0-3.5 and $(0.6-8.0) \times 10^{-7} \mathrm{erg} / \mathrm{s} / \mathrm{cm}^{2} / \mathrm{str}$, respectively.

When comparing the X-ray properties with the total integrated intensity in ${ }^{12} \mathrm{CO}$ $(J=1-0)$ observed by NANTENCO, we found a positive correlation between the X-ray intensity and ${ }^{12} \mathrm{CO}$ intensity and the fact that the photon index tends to be less steep when the ${ }^{12} \mathrm{CO}$ intensity increases. These trends suggest that an interaction between the ISM and shocks affects the process of the particle acceleration.

\section{References}

Sano, H. et al. 2015, ApJ, 799, 175S

Bamba, A., et al. 2004, ApJ, 602, 257

The H. E. S. S. Collaboration 2015, Science, 347, 406 